\title{
Produção de mudas de manjericão (Ocimum basilicum L.) sob efeito de diferentes
}

\section{substratos}

\author{
Production of basil (Ocimum basilicum L.) seedlings under different substrates \\ Producción de plántulas de albahaca (Ocimum basilicum L.) bajo diferentes sustratos
}

Thatiane Nepomuceno Alves

ORCID: https://orcid.org/0000-0002-9077-9104

Universidade Estadual Paulista, Brasil

E-mail: nepomuceno.alves@unesp.com

Beatriz Lívero Carvalho

ORCID: https://orcid.org/0000-0001-8433-2608

Universidade Estadual Paulista, Brasil

E-mail: beatriz.livero@unesp.br

Politon Thiago Pereira Guedes

ORCID: https://orcid.org/0000-0001-7156-4653

Universidade Estadual Paulista, Brasil

E-mail: politon.guedes@unesp.br

Nicholas Taborda Nordi

ORCID: https://orcid.org/0000-0001-8931-1777

Universidade Estadual Paulista, Brasil

E-mail: nicholasnordi@hotmail.com

Eduardo Santana Aires

ORCID: https://orcid.org/0000-0002-9765-3395

Universidade Estadual Paulista, Brasil E-mail: e.aires@unesp.br

Marcelo Munhoz Venâncio de Oliveira ORCID: https://orcid.org/0000-0002-0701-9623 Instituto Agronômico, Brasil

E-mail: marcelomunhozagro@gmail.com

Elizabeth Orika Ono

ORCID: https://orcid.org/0000-0001-7965-4696

Universidade Estadual Paulista, Brasil

E-mail: elizabeth.o.ono@unesp.br

João Domingos Rodrigues

ORCID: https://orcid.org/0000 -0003-4947-0997

Universidade Estadual Paulista, Brasil

E-mail: joao.domingos@unesp.br

\begin{abstract}
Resumo
O objetivo do estudo foi avaliar os efeitos de diferentes substratos na produção de mudas de cultivares de manjericão (Ocimum basicilicum L.). O delineamento experimental foi o inteiramente casualizado, com cinco tipos de substratos $\left(\right.$ Carolina $^{\circledR}$, Provaso ${ }^{\circledR}$, Provaso ${ }^{\circledR}+$ Fibra de coco, Fibra de coco + Turfa e Fibra de coco), sendo estudado em duas cultivares, Basilicão (BS) e Grecco a Palla (GP), com quatro repetições. As avaliações foram realizadas a partir do quinto dia após a emergência. Foi calculado o índice de velocidade de emergência (IVE) e percentual de emergência (E\%). A avaliação final foi aos 30 dias após a semeadura (DAS), sendo analisadas as seguintes variáveis: diâmetro do colo $(\mathrm{cm})$, altura da planta $(\mathrm{cm})$, comprimento de raiz $(\mathrm{cm})$, massa seca da parte aérea $(\mathrm{g})$, massa seca de raízes $(\mathrm{g})$, número de folhas, área foliar $\left(\mathrm{cm}^{2}\right)$ e índice de qualidade de Dickson (IQD). Observou-se que para as duas cultivares BS e GP o substrato Carolina ${ }^{\circledR}$ foi o que proporcionou maior porcentagem de emergência, assim como o menor tempo médio de emergência. Para altura, diâmetro do coleto, número de folhas e área foliar, observou-se, nas duas cultivares, que os substratos, Fibra de $\operatorname{coco}^{\circledR}$ e Fibra de coco + Turfa foram menores. Para comprimento de raiz, Fibra de coco foi superior para BS e Fibra de coco + turfa para GP. Levando em consideração todas as características morfológicas analisadas, na cv. BS o Provaso ${ }^{\circledR}$ e Fibra de coco foram os melhores; para a cv. GP foi o Fibra de coco + turfa.
\end{abstract}

Palavras-chave: Emergência; Qualidade de muda; Ocimum basilicum.

\footnotetext{
Abstract

The aim of the study was to evaluate the effects of different substrates on the production of seedlings of basil (Ocimum basicilicum L.). The experimental design was completely randomized, with five types of substrates
} 
(Carolina ${ }^{\circledR}$, Provaso ${ }^{\circledR}$, Provaso ${ }^{\circledR}+$ Coconut fiber, Coconut fiber + Peat and Coconut fiber), being studied in two cultivars, Basilicão (BS) and Grecco a Palla (GP), with four replications. The evaluations were carried out from the fifth day after the emergency. The emergency speed index (IVE) and emergency percentage (E\%) were calculated. The final evaluation was at 30 days after sowing (DAS), being analyzed the following variables: neck diameter (cm), plant height $(\mathrm{cm})$, root length $(\mathrm{cm})$, dry mass of the aerial part $(\mathrm{g})$, dry root mass $(\mathrm{g})$, number of leaves, leaf area $\left(\mathrm{cm}^{2}\right)$ and quality index ofDickson (IQD). It was observed that, for the two BS and GP cultivars, the Carolina ${ }^{\circledR}$ substrate was the one that provided the highest percentage of emergence, as well as the lowest mean time of emergence. For height, stem diameter, number of leaves and leaf area, it was observed in both cultivars that the substrates, Coco fiber ${ }^{\circledR}$ and Coco fiber + Peat, were smaller. For root length, coconut fiber was superior for BS and coconut fiber + peat for GP. Taking into account all the morphological characteristics analyzed, in cv. BS Provaso® and Coconut fiber were the best for cv. GP was the coconut fiber + peat.

Keywords: Emergence; Seedling quality; Ocimum basilicum.

\section{Resumen}

El objetivo del estudio fue evaluar los efectos de diferentes sustratos en la producción de plántulas de albahaca (Ocimum basicilicum L.). El diseño experimental fue completamente al azar, con cinco tipos de sustratos (Carolina ${ }^{\circledR}$, Provaso ${ }^{\circledR}$, Provaso® + fibra de coco, fibra de coco + fibra de turba y coco), en estudio en dos cultivares, Basilicão (BS) y Grecco a Palla (GP), con cuatro repeticiones.Las evaluaciones se realizaron a partir del quinto día posterior a la emergencia. Se calculó el índice de velocidad de emergencia (IVE) y el porcentaje de emergencia (E\%). La evaluación final fue a los 30 días después de la siembra (DAS), analizándose las siguientes variables: diámetro del cuello $(\mathrm{cm})$, altura de la planta $(\mathrm{cm})$, largo de la raíz $(\mathrm{cm})$, masa seca de la parte aérea $(\mathrm{g})$, masa seca de la raíz. (g), número de hojas, área foliar $\left(\mathrm{cm}^{2}\right)$ e índice de calidad deDickson (IQD). Se observó que, para los dos cultivares BS y GP, el sustrato Carolina ${ }^{\circledR}$ fue el que proporcionó el mayor porcentaje de emergencia, así como el menor tiempo medio de emergencia. Para altura, diámetro de tallo, número de hojas y área foliar, se observó en ambos cultivares que los sustratos, fibra de coco® y fibra de coco + turba, fueron menores. Para la longitud de la raíz, la fibra de coco fue superior para BS y fibra de coco + turba para GP. Teniendo en cuenta todas las características morfológicas analizadas, en el cv. BS Provaso® y la fibra de coco fueron los mejores para el cv. GP fue la fibra de coco + turba.

Palabras clave: Emergência; Calidad de la plântula; Ocimum basilicum.

\section{Introduçãa}

O manjericão (Ocimum basicilicum L.) é uma planta pertencente à família Lamiaceae e, no Brasil, foi introduzido pela colônia italiana sendo popularmente conhecida como manjericão comum, alfavaca, basilicão, erva-real, entre outras (Blank et al., 2004). É uma planta aromática e seu aroma pode ser adocicado, de canela, limão, cânfora, cravo e anis, dependendo da espécie utilizada (Almeida, 2011).

Classificado como um subarbusto é uma planta perene que se desenvolve melhor em regiões de clima quente. Já em regiões de temperaturas amenas, o seu ciclo de vida torna-se anual. Sua altura atinge de 30 a $50 \mathrm{~cm}$, seu plantio pode ser realizado durante todo o ano, podendo ser feito por estaquia ou sementes (Resende, 2010).

No Brasil, seu cultivo é realizado, principalmente, no estado de Sergipe, pois possui clima favorável à cultura (Marques et al., 2015), sendo o seu cultivo realizado, principalmente, pela agricultura familiar e sua comercialização é realizada por folhas verdes e frescas.

A propagação do manjericão pode ser tanto de forma sexuada como assexuada, sendo a propagação de mudas por meio de sementes rápida, em menos de quinze dias as sementes viáveis germinam. Apesar de ser um método bastante utilizado, sofre perdas de características genéticas desejáveis (Blank et al., 2014).

Para a propagação sexuada, o substrato tem função importante e é um dos fatores primordiais na produção de mudas, pois é o responsável pela retenção de água, oferta de nutrientes e influencia diretamente na germinação e formação das mudas. Além disso, também influencia no desenvolvimento do sistema radicular que precisa de boa formação para que as mudas sejam viáveis para serem levadas ao campo (Marques et al., 2017). O uso de mudas com maior vigor resulta em plantas mais produtivas (Costa et al., 2015).

A produção de mudas é uma das etapas mais importantes do sistema produtivo de hortaliças, que necessita da utilização de insumos de alta qualidade, sendo o substrato o de maior importância (Cardoso et al., 2017). Após a seleção de um 
substrato, vários fatores devem ser levados em consideração para a produção de uma boa muda, sendo estes: profundidade da semeadura, sementes de qualidade e manejo de irrigação (Dutra et al., 2016).

Atualmente, existem diversos tipos de substratos a serem utilizados na produção de mudas de manjericão, sejam eles comerciais como também os considerados caseiros, por isso deve-se conhecer a formulação de cada um, além de sua influência na produção de mudas. Nesse contexto, justifica-se avaliar os efeitos de diferentes substratos na produção de mudas de cultivares de manjericão (Ocimum basicilicum L.).

\section{Metodologia}

O experimento foi conduzido em casa de vegetação no período de outubro a novembro de 2019, no Departamento de Ciências Florestais, da Faculdade de Ciências Agronômicas (FCA), Universidade Estadual Paulista (UNESP), localizada no município de Botucatu-SP. A região, segundo classificação de Köppen, possui clima temperado quente (mesotérmico) úmido. Com chuvas no verão e seca no inverno, temperatura média do mês mais frio inferior a $17^{\circ} \mathrm{C}$ e do mês mais quente superior a $23^{\circ} \mathrm{C}$, com precipitações mensais superiores a $30 \mathrm{~mm}$ (Cunha \& Martins, 2009).

O delineamento experimental utilizado foi o inteiramente casualizado, com cinco tratamentos, tipos de substratos: Carolina $^{\circledR}$, Provaso ${ }^{\circledR}$, Provaso $^{\circledR}+$ Fibra de coco, Fibra de coco + Turfa e Fibra de coco, sendo estudado em duas cultivares, Basilicão (BS) e Grecco a Palla (GP), com quatro repetições, sendo utilizadas 36 plantas por parcela para cada cultivar analisada. As mudas foram produzidas em bandejas de polipropileno com 162 células, mantendo-se quatro linhas com nove células, para cada parcela, seguidas de uma linha vazia. Das quatro linhas, a primeira e a última, serviram de bordadura.

A semeadura foi realizada colocando-se duas sementes por célula e as avaliações foram realizadas a partir do quinto dia após a emergência (DAE), sendo avaliado o número de sementes emergidas, para o cálculo do tempo médio de emergência (TME) e o percentual de emergência (E\%). Foram consideradas sementes emergidas àquelas que emitiram o primeiro par de folhas cotiledonares. A partir desses dados, determinou-se o percentual de emergência e o índice de velocidade de emergência, conforme fórmula proposta por Maguire (1962): em que IVE $=(\mathrm{E} 1 / \mathrm{N} 1)+(\mathrm{E} 2 / \mathrm{N} 2)+\ldots+(\mathrm{En} / \mathrm{Nn})$, sendo E=número de plântulas emergidas e $\mathrm{N}=$ número de dias após a semeadura.

A avaliação final foi realizada aos 30 dias após a semeadura (DAS), sendo analisadas as seguintes variáveis nas plântulas: diâmetro do colo $(\mathrm{cm})$, altura da planta $(\mathrm{cm})$, comprimento de raiz $(\mathrm{cm})$, massa seca da parte aérea $(\mathrm{g})$, massa seca de raízes (g), número de folhas, área foliar $\left(\mathrm{cm}^{2}\right)$ e índice de qualidade de Dickson (IQD).

As plântulas foram separadas em folha, caule e raiz e acondicionadas em sacos de papel para secagem em estufa de circulação forçada de ar por 72 horas a temperatura de $65^{\circ} \mathrm{C}$. Em seguida, foram pesadas em balança analítica de precisão $(0,001 \mathrm{~g})$ e obtidas a matéria seca da parte aérea e de raízes, sendo expressas em g.planta-1. A área foliar (AF) foi obtida em integralizador de área em $\mathrm{cm}^{2}$ (Area Meter da LI-COR®).

O IQD foi determinado em função da altura da planta $(\mathrm{H})$, diâmetro do colo (DC), massa seca da parte aérea (MSPA) e massa seca da raiz (MSR), por meio da seguinte fórmula (Dickson et al., 1960):

$$
I Q D=\frac{M 5 T(g)}{\frac{H(\mathrm{~cm} !)}{D C(\mathrm{~mm})}+\frac{M S P A(g)}{M S R(g)}}
$$

Os dados foram submetidos à análise de variância e as médias comparadas pelo teste Tukey ao nível de $5 \%$ de probabilidade (Ferreira, 2014). 


\section{Resultados e Discussão}

A partir da análise de variância foi possível verificar que houve diferença para todas as variáveis analisadas: porcentagem de emergência (E\%) e tempo médio de emergência (TME), altura da planta (AP), diâmetro do coleto (DC), comprimento de raiz (CR), número de folhas (NF), área foliar (AF), índice de qualidade de Dickson (IQD), massa seca da raiz (MSR) e massa seca da parte aérea (MSPA).

Para valores médios de emergência na cultivar BS, observa-se que o substrato Carolina ${ }^{\circledR}$ promoveu a maior porcentagem de emergência e menor tempo médio de emergência (Tabela 1). A formulação do substrato Carolina ${ }^{\square}$, em sua composição, contem esfagno e turfa, o que favorece boa retenção de água e o nível adequado de hidratação é de fundamental importância, pois nessa condição, os processos metabólicos das sementes são ativados. Esse fato resulta no desenvolvimento satisfatório do eixo embrionário, assim como na emergência das plântulas (Silva et al., 2011; Costa et al., 2015). A densidade e porosidade do substrato Carolina $^{\circledR}$, possivelmente, facilitou a emergência da radícula pelo substrato, levando à protusão da raiz, rapidamente.

Tabela 1. Valores médios de porcentagem de emergência ( $\mathrm{E} \%$ ) e tempo médio de emergência (TME) de sementes de manjericão cv. Basilicão (BS) em função de diferentes substratos. Botucatu-SP, 2019.

\begin{tabular}{lcccccc}
\hline Tratamentos & Carolina $^{\circledR}$ & Provaso $^{\circledR}$ & $\begin{array}{c}\text { Provaso }^{\circledR}+ \\
\text { Fibra de coco }^{2}\end{array}$ & Fibra de coco & $\begin{array}{c}\text { Fibra de coco } \\
+ \text { Turfa }\end{array}$ & C.V. (\%) \\
\hline E\% & BS \\
TME (dias) & $37,50 \mathrm{a}$ & $87,50 \mathrm{ab}$ & $67,50 \mathrm{bc}$ & $62,50 \mathrm{c}$ & $70,00 \mathrm{bc}$ & 13,41 \\
\hline
\end{tabular}

Médias seguidas de mesma letra minúscula na linha não diferem estatisticamente entre si pelo teste Tukey $(\mathrm{p}<0,05)$. Fonte: Autores.

Para altura, diâmetro do coleto, número de folhas e área foliar observou-se para a cv. BS, que os substratos, Fibra de coco e Fibra de coco + Turfa resultaram nos menores valores, os demais substratos apresentaram valores superiores e não diferiram entre si (Tabela 2). Dentre as características morfológicas, a altura da planta e diâmetro do coleto são um dos parâmetros mais importantes para se estimar o crescimento das mudas (Silva et al., 2011; Souza et al., 2013)

Tabela 2. Valores médios de altura (AP), diâmetro do coleto (DC), comprimento de raiz (CR), número de folhas (NF), área foliar (AF), índice de qualidade de Dickson (IQD), massa seca da raiz (MSR) e massa seca da parte aérea (MSPA) de mudas de manjericão cv. Basilicão (BS) em função de diferentes substratos. Botucatu-SP, 2019.

\begin{tabular}{lllllllll}
\hline \multirow{1}{*}{$\begin{array}{c}\text { BS } \\
\text { Tratamentos }\end{array}$} & AP & DC & CR & NF & AF cm ${ }^{2}$ & IQD & MSR g & MSPA g \\
planta & Cm & Mm & Cm & & & & \\
\cline { 2 - 10 } Carolina ${ }^{\circledR}$ & $14,0 \mathrm{a}$ & $3,3 \mathrm{a}$ & $9,3 \mathrm{bc}$ & $6,0 \mathrm{a}$ & $41,3 \mathrm{a}$ & $0,08 \mathrm{~b}$ & $0,18 \mathrm{c}$ & $0,25 \mathrm{~b}$ \\
Provaso ${ }^{\circledR}$ & $12,0 \mathrm{a}$ & $3,2 \mathrm{a}$ & $10,5 \mathrm{ab}$ & $6,0 \mathrm{a}$ & $39,4 \mathrm{a}$ & $0,15 \mathrm{a}$ & $0,33 \mathrm{a}$ & $0,43 \mathrm{a}$ \\
Provaso ${ }^{\circledR}+$ Fibra de coco & $11,8 \mathrm{a}$ & $3,3 \mathrm{a}$ & $8,8 \mathrm{bc}$ & $6,0 \mathrm{a}$ & $38,8 \mathrm{a}$ & $0,07 \mathrm{~b}$ & $0,14 \mathrm{~d}$ & $0,25 \mathrm{~b}$ \\
Fibra de coco & $5,0 \mathrm{~b}$ & $2,9 \mathrm{~b}$ & $12,5 \mathrm{a}$ & $5,0 \mathrm{~b}$ & $25,4 \mathrm{~b}$ & $0,14 \mathrm{a}$ & $0,19 \mathrm{~b}$ & $0,10 \mathrm{c}$ \\
Fibra de coco + Turfa & $6,9 \mathrm{~b}$ & $2,9 \mathrm{~b}$ & $7,9 \mathrm{c}$ & $5,0 \mathrm{~b}$ & $25,9 \mathrm{~b}$ & $0,07 \mathrm{~b}$ & $0,14 \mathrm{e}$ & $0,08 \mathrm{~d}$ \\
\hline CV\% & 10,86 & 1,77 & 12,1 & 0 & 8,42 & 17,62 & 0,32 & 0,96 \\
\hline
\end{tabular}

Médias seguidas de mesma letra minúscula nas colunas, não diferem estatisticamente entre si pelo teste Tukey a 5\% de probabilidade. Fonte: Autores. 
O substrato Fibra de coco foi o que proporcionou o maior comprimento de raiz, em média $12,5 \mathrm{~cm}$, diferindo estatisticamente dos demais, com exceção do Provaso ${ }^{\circledR}$, que diferiu apenas da mistura de Fibra de coco + Turfa (Tabela 2).

Nas variáveis, índice de qualidade de Dickson, o substrato Provaso ${ }^{\circledR}$ e Fibra de coco foram aqueles que apresentaram os melhores resultados quando comparados aos demais substratos para a cultivar BS (Tabela 2). Para massa seca de raiz e de parte aérea, o Provaso ${ }^{\circledR}$ diferiu significativamente dos demais substratos. O acúmulo de matéria seca na planta está relacionado com a produção de fotoassimilados, sendo que para essas condições é necessário que as plântulas estejam em ótimas condições de luminosidade, nutrição, disponibilidade hídrica, etc. (Silva et al., 2019). Assim, as mudas terão condições para sobreviver, após o transplantio, e apresentar posteriormente desempenho produtivo no campo.

Tabela 3. Valores médios de porcentagem de emergência (G\%) e tempo médio de emergência (TMG) de sementes da cultivar de manjericão Grecco a Palla (GP) em função de diferentes substratos. Botucatu-SP, 2019.

\begin{tabular}{lcccccc}
\hline Tratamentos & Carolina $^{\circledR}$ & Provaso $^{\circledR}$ & $\begin{array}{c}\text { Provaso }^{\circledR}+ \\
\text { Fibra de coco }\end{array}$ & Fibra de coco & $\begin{array}{c}\text { Fibra de coco } \\
+ \text { Turfa }\end{array}$ & C.V. (\%) \\
\hline E\% & GP & & & \\
TMG & $98,75 \mathrm{a}$ & $64,50 \mathrm{~b}$ & $67,50 \mathrm{~b}$ & $60,00 \mathrm{~b}$ & $60,00 \mathrm{~b}$ & 9,52 \\
\hline
\end{tabular}

Médias seguidas de mesma letra minúscula na linha não diferem estatisticamente entre si pelo teste Tukey $(\mathrm{p}<0,05)$. Fonte: Autores.

Para a cultivar GP, o substrato Carolina ${ }^{\square}$ diferiu dos demais, apresentando melhor resposta quanto à porcentagem de emergência. Isso pode ser explicado pela presença de vermiculita que, segundo Ramos et al. (2003), devido à sua porosidade, apresenta boa relação água/ar. Rodrigues et al. (2013) ao avaliarem a taxa de germinação de sementes de manjericão em substratos alternativos obtiveram melhor resultado com o uso de fibra de coco, com percentagem de germinação superior a $95 \%$.

Com relação ao tempo médio de emergência (TME) deve ser analisado, qual substrato apresentou menor valor, ou seja, em qual substrato que a semente germinou mais rapidamente. Assim, observou-se que para a cultivar GP os substratos Carolina $^{\circledR}$ e Provaso ${ }^{\circledR}$ + Fibra de coco foram aqueles que promoveram a germinação em menor tempo.

A densidade e porosidade do substrato Carolina ${ }^{\circledR}$, possivelmente, facilitou a emergência da radícula pelo substrato, levando a protrusão da raiz, rapidamente. Para essa avaliação, os substratos Carolina ${ }^{\circledR}$ e Provaso $^{\circledR}$ seriam os mais indicados para acelerar a germinação de sementes, uma vez que, o composto presente nesses substratos auxilia na capacidade de armazenamento de água, melhora a drenagem e a aeração do solo, além de manter o pH estável. Bassaco et al. (2014) realizaram estudos em que a utilização do substrato reduziu o tempo de germinação, principalmente, em temperaturas entre 25$30^{\circ} \mathrm{C}$.

Quanto à altura de plantas para a cultivar GP o substrato Provaso ${ }^{\square}$ apresentou melhor desempenho, seguido dos substratos Carolina $^{\square}$ e Provaso ${ }^{\square}+$ Fibra de Coco (Tabela 4). Estes mesmos substratos além de se destacarem na altura da planta, também apresentaram resultados positivos para diâmetro do coleto e os substratos citados proporcionaram mudas mais vigorosas de manjericão, pois incrementaram o diâmetro do colo e a altura das plantas. Dentre as características morfológicas, a altura da planta e diâmetro do colo são um dos parâmetros mais importantes para se estimar o crescimento das mudas (Souza et al., 2013). 
Tabela 4. Valores médios de altura da planta (AP), diâmetro do coleto (DC), comprimento de raiz (CR), número de folhas (NF), área foliar (AF), índice de qualidade de Dickson (IQD), massa seca da raiz (MSR) e massa seca da parte aérea (MSPA) de mudas de manjericão cv. Grecco a Palla (GP) em função de diferentes substratos. Botucatu-SP, 2019.

\begin{tabular}{|c|c|c|c|c|c|c|c|c|}
\hline $\begin{array}{c}\text { GP } \\
\text { Tratamentos }\end{array}$ & $\begin{array}{l}\mathrm{AP} \\
\mathrm{cm}\end{array}$ & $\begin{array}{l}\mathrm{DC} \\
\mathrm{Mm}\end{array}$ & $\begin{array}{l}\mathrm{CR} \\
\mathrm{Cm}\end{array}$ & NF & $\begin{array}{l}\mathrm{AF} \mathrm{cm}^{2} \\
\text { planta }\end{array}$ & IQD & MSR g & MSPA $g$ \\
\hline \multicolumn{9}{|c|}{ GP } \\
\hline Carolina ${ }^{\circledR}$ & $9,3 \mathrm{ab}$ & $3,1 \mathrm{a}$ & $10,3 \mathrm{abc}$ & $12,0 \mathrm{a}$ & $23,4 \mathrm{a}$ & $0,061 \mathrm{c}$ & $0,09 \mathrm{c}$ & $0,25 \mathrm{a}$ \\
\hline Provaso® & $10,3 \mathrm{a}$ & $3,0 \mathrm{a}$ & $12,0 \mathrm{ab}$ & $12,0 \mathrm{a}$ & $23,6 \mathrm{a}$ & $0,045 \mathrm{~b}$ & $0,12 \mathrm{~b}$ & $0,22 \mathrm{c}$ \\
\hline Provaso $®$ + Fibra de coco & $9,9 \mathrm{ab}$ & $2,3 \mathrm{~b}$ & $7,5 \mathrm{c}$ & $12,0 \mathrm{a}$ & $23,4 \mathrm{a}$ & $0,054 \mathrm{~d}$ & $0,08 \mathrm{~d}$ & $0,23 \mathrm{~b}$ \\
\hline Fibra de coco & $7,5 \mathrm{~b}$ & $2,3 \mathrm{~b}$ & $8,8 \mathrm{bc}$ & $10,0 \mathrm{c}$ & $15,7 \mathrm{~b}$ & $0,045 \mathrm{e}$ & $0,09 \mathrm{c}$ & $0,053 \mathrm{e}$ \\
\hline Fibra de coco + Turfa & $4,8 \mathrm{c}$ & $2,2 \mathrm{~b}$ & $12,3 \mathrm{a}$ & $11,0 \mathrm{~b}$ & $17,5 \mathrm{~b}$ & $0,091 \mathrm{a}$ & $0,14 \mathrm{a}$ & $0,062 \mathrm{~d}$ \\
\hline $\mathrm{CV} \%$ & 13,84 & 4,02 & 14,25 & 2,25 & 5,7 & 1,78 & 1,62 & 0,95 \\
\hline
\end{tabular}

Médias seguidas de mesma letra minúscula nas colunas, não diferem estatisticamente entre si pelo teste Tukey a 5\% de probabilidade. Fonte: Autores.

Segundo Paulus et al. (2011), levando em consideração os fatores importantes a serem observados no processo de produção de mudas de boa qualidade, os substratos representam fator primordial. Daniel et al. (1997) mostraram resultados em que substratos auxiliaram no crescimento em diâmetro de caule e que essa característica é importante para a sobrevivência da muda no campo. Em consideração à esse aspecto é necessário utilizar substratos que apresentem bons resultados para essa característica.

Para comprimento de raiz (Tabela 4), o substrato Fibra de Coco + Turfa apresentou os melhores resultados, sendo o responsável pelo maior crescimento da raiz, $12,3 \mathrm{~cm}$, seguido dos substratos Provaso ${ }^{\square}$ que apresentou valor médio de $12 \mathrm{~cm}$ e Carolina $^{\square}$, com valor de $10,3 \mathrm{~cm}$.

Em relação ao número de folhas (Tabela 4), o substrato Provaso ${ }^{\circledR}+$ Fibra de coco apresentou maior número de folhas, diferindo estatisticamente dos demais substratos. O substrato Carolina ${ }^{\circledR}$ e Provaso ${ }^{\circledR}$ não diferiram entre si, enquanto Fibra de coco + Turfa e Fibra de coco apresentaram as menores médias, sendo diferentes entre si. A mistura de Provaso ${ }^{\circledR}+$ Fibra de Coco foi o substrato com melhor resposta, quanto ao número de folhas.

Em estudo utilizando a mistura de substratos realizado por Gazola et al. (2015) mostraram que a utilização de Fibra de coco com substratos comerciais foi uma alternativa importante na produção de mudas de alface (Lactuca sativa L.), pois, essa mistura além de diminuir o uso do produto comercial, potencializa as características que são desejadas para mudas. Além disso, Mendonça et al. (2007) observaram que os melhores resultados para número de folhas foram aqueles que apresentavam algum tipo de matéria orgânica em sua mistura.

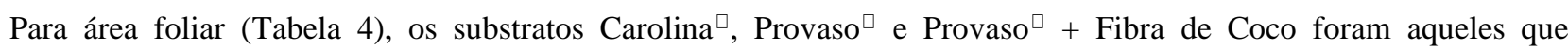
apresentaram melhores resultados para a cultivar GP, mostrando que auxiliam positivamente no aumento da área foliar da cultivar estudada. Essa característica é extremamente importante, pois interfere diretamente na capacidade da planta em interceptar radiações luminosas e realizar fotossíntese, assim, sendo indispensável para o rendimento da cultura (Figueiredo et al., 2010). Carmo et al. (2018) utilizaram substratos orgânicos na produção de mudas e observaram que estes auxiliaram no aumento da área foliar, principalmente, aqueles que continham cama de frango em sua composição.

Para as variáveis índice de qualidade de Dickson e massa seca da raiz (Tabela 4), o substrato Fibra de Coco + Turfa diferiu dos demais substratos e apresentou melhor resultado. De acordo com Fonseca et al. (2002), o índice de qualidade de Dickson (IQD) é um bom indicador de qualidade, pois leva-se em consideração o equilíbrio da distribuição da biomassa, mostrando-se um importante parâmetro a ser empregado na avaliação da qualidade de mudas. 
Em relação à massa seca da parte aérea, diferente da massa seca de raiz, o substrato que se destacou e apresentou os melhores resultados foi o Carolina ${ }^{\square}$ e o substrato Fibra de Coco apresentou o menor resultado nessa variável.

\section{Conclusão}

O substrato Carolina ${ }^{\circledR}$ nas duas cultivares de manjericão foi o que apresentou maior percentual de emergência e menor tempo médio de emergência.

Levando em consideração todas as características morfológicas analisadas, para o índice de qualidade de mudas, que indica o melhor substrato, na cv. BS o Provaso ${ }^{\circledR}$ e Fibra de coco foram aqueles que apresentaram os melhores resultados e para a cv. GP, Fibra d coco + turfa apresentou o melhor índice.

\section{Referências}

Almeida, Z. M. de. (2011) Plantas medicinais. EDUFBA.

Bassaco, M. V., Nogueira, A. C., \& Cosmo, N. L. (2014). Avaliação da germinação em diferentes temperaturas e substratos e morfologia do fruto, semente e plântula de Sebastiana brasiliensis. Floresta, 44 (3),381-392.

Blank, A. F., Arrigoni-Blank, M. D. F., Carvalho Filho, J. L. S. D., Santos Neto, A. L. D., \& Amancio, V. F. (2014). Produção de Mudas de Manjericão com Diferentes Tipos de Substratos e Recepientes. Biosci, 30 (1),39-44.

Blank, M. F., Silva-Mann, R., \& Mendonça, M. C. Caracterização morfológica e agronômica de acessos de manejericão e alfavaca. (2004) Horticultura Brasileira, 22, (1) 113-116.

Cardoso, A. I. I., Piedade, M. B. S., Rodrigues, J. M., \& Ricardo, L. E. (2017). Produção de couve chinesa em função da fertirrigação nitrogenada e potássica nas mudas. Horticultura Brasileira, 35 (4) 512-518.

Carmo, M. C., Santos, W. P., Machado, C. M., Santos, M. R. G., \& Bravin, N. P. (2018). Diferentes substratos orgânicos na produção de mudas de berinjela na Amazônia Ocidental. Agrarian Academy, 5(9), 188-196.

Costa, L. A. D. M., Costa, M. S. S. M., Pereira, D. C., Bernardi, F. H., \& Maccari, S. (2015). Avaliação de substratos para a produção de mudas de tomate e pepino. Revista Ceres, 60, (5) 675-682.

Cunha, A. R., \& Martins, D. (2009). Classificação climática para os municípios de Botucatu e São Manuel, SP. Irriga, 14(1), 1-11

Daniel, O. A. C. T., Vitorino, A. C. T., Alovisi, A. A., Mazzochin, L., Tokura, A. M., Pinheiro, E. R., \& Souza, E. D. (1997) Aplicação de fósforo em mudas de Acacia mangium Willd. Revista Árvore, 21(2), 163-168.

Dickson, A., Leaf, A. L., \& Hosner, J. F. (1960). Quality appraisal of white spruce and white pine seedling stock in nurseries. Forestry Chronicle, 36(1), 1013 .

Dutra, A. F., Araújo, M. M., Rorato, D. G., \& Mieth, P. (2016) Germinação de sementes e emergência de plântulas de Luehea divaricata Mart. et. Zucc. em diferentes substratos. Ciência Florestal, 26(2), 411-418.

Figueiredo, R. T., Guiscem, J. M., Chaves, A. M. S., Aguiar Junior, R. A., Silva, A. G. P., Paiva, J. B. P., \& Santos, F. N. (2010). Relação entre a área foliar, número de folhas e biomassa seca e fresca da planta de rúcula. Horticultura brasileira, 28(2), 913-918.

Gazola, T., Gualberto, R., Dias, M. F., Cipola Filho, M. L., Belapart, D., \& de Castro, E. B. (2015). Avaliação de substratos alternativos na produção de mudas e desenvolvimento de plantas de alface. Unimar Ciência, 24(1), 33-39.

Maguire, J. D. (1962). Speed of germination-aid in selection and evaluation for seedling emergence and vigor. Crop Science, 2 (1), $176-177$.

Marques, L. O. D., Mello-Farias, P., de Lima, A. Y. B., Malgarim, M. B., \& dos Santos, R. F. (2017). Desempenho de diferentes substratos e influência do frio na germinação de sementes de araçá amarelo. Revista da Jornada da Pós Graduação e Pesquisa, 14(1), 1169-1180.

Marques, P. A. A., José, J. V., Rocha, H. S. da, Fraga J, E. F., Soares, D. A., Duarte, S. N. (2015). Consumo hídrico do manjericão por meio de lisímetro de drenagem. Irriga, 20 (4), 745-761.

Mendonça, V., De Abreu, N. A. A., De Souza, H. A., Ferreira, E. A., \& Ramos, J. D. (2007) Diferentes níveis de composto orgânico na formulação de substrato para a produção de mudas de mamoeiro 'Formosa'. Caatinga, 20 (1), 49-53.

Paulus, D., Valmorbida, R., Toffoli, E., Paulus, E., \& Garlet, T. (2011). Avaliação de substratos orgânicos na produção de mudas de hortelã (Mentha gracilis R. Br. e Mentha x villosa Huds.). Revista. Brasileira de plantas medicinais.13 (1), 90-97.

Ramos, J. D., Matos, L. E. S., Gontijo, T. C. A., Pio, R., Junqueira, K. P., \& Santos, F. C. (2003). Enraizamento de estacas herbáceas de ‘’Mirabolano (Prunus cerasifera) em diferentes substratos e concentrações de ácido indolbutírico. Revista Brasileira de Fruticultura, 25 (1), $189-191$. 
Research, Society and Development, v. 10, n. 2, e58210212867, 2021

(CC BY 4.0) | ISSN 2525-3409 | DOI: http://dx.doi.org/10.33448/rsd-v10i2.12867

Resende, R. F. Produção de biomassa e óleo essencial de Manjericão (Ocimum basilicum L.) em diferentes épocas, ambientes de cultivo e tipos de adubação. (2010). Dissertação (Mestrado) - Curso de Agronomia, Universidade Federal de Uberlândia, Uberlândia.

Rodrigues, A. A. J., Araújo Torres, R., de Oliveira Santos, E., de Lima, L. F., \& Takane, R. J. (2013). Avaliação de substratos alternativos na germinação de sementes de manjericão (Ocimum basilicum L.). Cadernos de Agroecologia, 8 (2).

Silva, J. D. C., Leal, T. T. B., Araújo, R. M., Gomes, R. L. F., Araújo, A. S. F., Melo, W. J. (2011). Emergência e crescimento inicial de plântulas de pimenta ornamental e celosia em substrato à base de composto de lodo de curtume. Ciência Rural, 41 (3), 412-417.

Silva, L. P., Oliveira, A. C., Alves, N. F., Silva, V. L., Silva, T. I. (2019). Uso de substratos alternativos na produção de mudas de pimenta e pimentão. In: Colloquium Agrariae. 15(3), 104-115.

Souza, E. G. F., Barros Júnior, A. P., Silveira, L. M. D., Santos, M. G. D., \& Silva, E. F. D. (2013) Emergência e desenvolvimento de mudas de tomate IPA 6 em substratos, contendo esterco ovino. Revista Ceres, 60 (4), 902-907. 